\title{
Prescription of antipsychotics in people with dementia ${ }^{\dagger}$
}

\author{
Clive Ballard, Anne Corbett and Robert Howard
}

\section{Summary}

Gerhard et al clarify our understanding of mortality associated with antipsychotic use in people with dementia, by demonstrating a clear dose relationship and highlighting key questions regarding the relative mortality risk of different atypical antipsychotics. The study also suggests that antipsychotics may confer risks of increased mortality in older people without dementia.

\section{Declaration of interest}

C.B. has received speaker fees and consultancy honoraria from Acadia, Lundbeck, Bristol-Myers Squibb, Napp, Takeda, Onsaka, Synexus and Novartis. A.C. has received honoraria from Bial, Lundbeck, Novartis and Acadia.
Clive Ballard (pictured) is Professor of Age-related Diseases and a consultant old age psychiatrist at King's College London and has led key clinical trials regarding antipsychotic use in dementia. Anne corbett is a lecturer in dementia communication at King's College London and has written key systematic reviews regarding antipsychotics and other dementia treatment issues. Robert Howard is Professor of Old Age Psychiatry at King's College London and has led pivotal clinical trials regarding dementia treatment.

More than $90 \%$ of patients with dementia will experience at least one behavioural and psychological symptom of dementia (BPSD) during the course of their condition. ${ }^{1}$ The most common BPSDs are delusions, hallucinations, agitation and aggression. They cause significant distress to people with dementia and their caregivers, increase the likelihood of institutionalisation and represent a considerable clinical challenge for clinicians. The management of BPSDs is extremely challenging. Best practice guides emphasise the assessment and treatment of contributing medical conditions, appropriate analgesia for pain relief and the efficacy of simple non-pharmacological interventions. ${ }^{2}$ Despite these measures, however, there remains an important role for pharmacological management.

\section{The challenge of treating BPSD}

It has been established through robust placebo-controlled clinical trials that the antipsychotics risperidone, olanzapine and aripiprazole confer modest but significant benefits in the treatment of aggression and psychosis over 6-12 weeks in people with Alzheimer's disease. ${ }^{1,3}$ Trials have also failed to demonstrate treatment benefits for quetiapine. ${ }^{1,3}$ There is very limited evidence regarding any treatment benefits in people with non-Alzheimer's dementia and it is far less clear whether there are any benefits of longer-term treatment, ${ }^{4}$ although evidence suggests significant continued benefit for at least 6 months in people with a clear initial positive treatment response. ${ }^{5}$

The modest advantages in the treatment of psychosis and aggression do however need to be balanced against numerous adverse events including Parkinsonism, sedation, gait disturbance, accelerated cognitive decline, pneumonia and thromboembolic events including stroke, ${ }^{1,3}$ and the overall impact of treatment may be detrimental with respect to quality of life even when symptoms improve. It is however the significant increase in mortality risk (1.5- to 1.8 -fold $)^{6}$ that has led to particular

'See pp. 44-51, this issue. concerns for regulators such as the US Food and Drug Administration, the European Medicines Agency and the UK Medicines and Healthcare products Regulatory Agency, all of whom have issued warnings regarding the mortality risk associated with the use of antipsychotics in people with dementia. The relative risk of mortality remains elevated with continued antipsychotic treatment and the actual number of attributable deaths therefore continues to increase with longer duration of treatment.

In a report for the Department of Health in 2009, Professor Sube Banerjee estimated that 180000 patients with dementia in the UK were being prescribed antipsychotic medication and that unnecessary prescription of antipsychotics to people with dementia leads to 1800 additional deaths per year. ${ }^{7}$ This triggered a government-driven imperative to reduce unnecessary prescribing of antipsychotics in people with dementia. Based on national audit data, this has resulted in a halving of the rates of antipsychotic prescribing. ${ }^{8}$ Most of the cohort studies informing risk and the double-blind placebo controlled trials examining the discontinuation of antipsychotics in people with dementia have been conducted in a prescribing environment where it has been estimated that two-thirds of prescriptions were unnecessary. The reduction in use of antipsychotic medication has probably been achieved through more judicious and better-focused prescribing. It is probable that individuals with dementia who are still prescribed antipsychotics have more severe and intractable BPSDs, and it is unclear how this more targeted use of antipsychotics has altered the potential benefit-to-risk ratio for patients prescribed these agents.

Despite the reductions in antipsychotic use, there are still a significant number of people with dementia prescribed antipsychotics and it is important that we continue to use research evidence to improve the safety and quality of prescribing. There are a number of key unanswered questions regarding the differential mortality risk of individual antipsychotic drugs, the relationship between dose and mortality, and individual patient factors which may predict or mediate risk. In addition, there has been very little research focus on whether antipsychotic medications also increase mortality in older people without dementia.

\section{Emerging data on mortality associated with antipsychotics}

The paper from Gerhard and colleagues ${ }^{9}$ addresses several of these key issues. The study examined 180-day non-cancer mortality in a 
large retrospective cohort of 136000 adults over the age of 65 living in the community and treated with antipsychotic medication. Approximately a third of the individuals had dementia and the other two-thirds did not. Patients with a diagnosis of schizophrenia, bipolar disorder or cancer during the 180-day period preceding the prescription of antipsychotics were excluded. As all patients included in the analysis were prescribed antipsychotic medication, the aim was to examine the specific risk of individual antipsychotics compared with risperidone, and to evaluate the relationship between mortality risk and dose. Inclusion of people with and without dementia also enabled the authors to comment on the broader mortality risk associated with antipsychotic prescribing.

When combining outcomes with all antipsychotics, there was a clear relationship between dose and mortality risk. When examining individual antipsychotics, the mortality risk was substantially increased for haloperidol and decreased for quetiapine compared with risperidone. After controlling for dose and other major confounding factors, the mortality risk, although reduced, was still significantly higher for haloperidol than for risperidone. Of particular note in this analysis, olanzapine, aripiprazole and quetiapine all had significantly reduced mortality risk compared with risperidone. The relative mortality risks of different agents and by dose were similar in patients with and without dementia.

\section{Implications for clinical practice and research}

The study is the first to carefully examine the relationship between antipsychotic dose and mortality risk, showing a clear stepwise increase in risk from low to medium and from medium to high doses. This is potentially extremely important in guiding clinical practice, although it is still important that treatments are prescribed within the therapeutic window shown to be effective. For example, the placebo-controlled randomised controlled trials of risperidone clearly show that a dose of $0.5 \mathrm{mg}$ daily does not confer benefit in the treatment of aggression or psychosis, and that $1 \mathrm{mg} /$ day is the lowest effective dose. ${ }^{3}$ Gerhard et al's study also confirms several other reports indicating that haloperidol is associated with higher mortality risk and quetiapine with lower mortality risk than risperidone in patients with dementia. ${ }^{10}$ Although the mortality risk is slightly lower for quetiapine, this is of limited clinical utility given the consistent evidence from randomised controlled trials in people with Alzheimer's disease and Parkinson's disease dementia that quetiapine is ineffective in treating psychosis, aggression or agitation. ${ }^{1,3}$ In addition, quetiapine appears to have a more substantial detrimental impact on cognition than other atypical antipsychotics in patients with Alzheimer's disease. Of more interest are the findings that olanzapine and aripiprazole may be associated with lower mortality risk than risperidone when adjusting for dose. The findings do however have to be interpreted cautiously as there were a smaller number of patients treated with these agents, particularly with aripiprazole. Of note, both drugs have been shown to have comparative efficacy to risperidone in previous meta-analyses of randomised controlled trials. ${ }^{3,4}$ A further metaanalysis of cohort studies reporting mortality associated with individual drugs is now urgently needed to determine whether olanzapine or aripiprazole could be an equally effective and slightly safer alternative to risperidone for the treatment of aggression and psychosis in patients with dementia.

What does the study tell us about the risks of antipsychotic use in older people without a dementia diagnosis - in particular those with functional psychoses for whom management with a non-drug treatment or alternative class of psychotropic would not be an option? Gerhard and colleagues acknowledge that dementia may have been underdiagnosed in their sample, but it is just as likely that patients with schizophrenia and bipolar disorder would also have been underdiagnosed and consequently would have been inadvertently included among the two-thirds of participants without a dementia diagnosis. In the absence of an untreated comparison group it is of course impossible to comment on the absolute increase in mortality associated with antipsychotic use in patients with functional psychoses. But the demonstration of a dose-response relationship with mortality risk that was independent of dementia diagnosis is strongly suggestive of similar risks to those seen in people with dementia. It would be important for Gerhard and colleagues to re-examine their data-set, looking specifically at patients who did have a diagnosis of schizophrenia or bipolar disorder to confirm this. It is probably already established practice to use the lowest effective antipsychotic dose and to avoid haloperidol for older patients with functional psychoses, and this study hints at the potential dangers of not following these principles.

\section{Acknowledgements}

The authors would like to thank the NIHR Mental Health Biomedical Research Centre and Dementia Unit at South London and Maudsley NHS Foundation Trust and (Institute of Psychiatry) King's College London in supporting their time in producing this editorial.

Clive Ballard, MD, MRCPsych, Anne Corbett, PhD, Wolfson Centre for Age-Related Diseases, King's College London; Robert Howard, MD, MRCPsych, Institute of Psychiatry, King's College London, UK

Correspondence: Clive Ballard, Professor of Age-Related Diseases, Wolfson Centre for Age-Related Diseases, Guy's Campus, King's College London, London SE1 1UL, UK. Email: clive.ballard@kcl.ac.uk

First received 29 Apr 2014, accepted 1 May 2014

\section{References}

1 Ballard CG, Gauthier S, Cummings JL, Brodaty H, Grossberg GT, Robert P et al. Management of agitation and aggression associated with Alzheimer disease. Nat Rev Neurosci 2009; 5: 245-55.

2 Alzheimer's Society. Optimising Treatment and Care for Behavioural and Psychological Symptoms of Dementia. Alzheimer's Society, 2013.

3 Ballard C, Howard R. Neuroleptic drugs in dementia: benefits and harm. Nat Rev Neurosci 2006; 7: 492-500.

4 Ballard C, Lana M, Theodoulou M, Douglas S, McShane R, Jacoby R, et al. A randomised, blinded, placebo-controlled trial in dementia patients continuing or stopping neuroleptics (the DART-AD trial). PLOS Med 2008; 5: 76.

5 Devanand DP, Mintzer J, Schultz SK, Andrews HF, Sultzer DL, de la Pena D, et al. Relapse risk after discontinuation of risperidone in Alzheimer's disease. N Engl J Med 2012; 367: 1497-507.

6 Schneider LS, Dagerman KS, Insel P. Risk of death with atypical antipsychotic drug treatment for dementia: meta-analysis of randomized placebocontrolled trials. JAMA 2005; 294: 1934-43.

7 Banerjee S. The Use of Antipsychotic Medication for People with Dementia: Time for Action. Department of Health, 2009.

8 Health and Social Care Information Centre. National Dementia \& Antipsychotic Prescribing Audit 2012. HSCIC, 2012.

9 Gerhard T, Huybrechts K, Olfson M, Schneeweiss S, Bobo WV, Doraiswamy PM, et al. Comparative mortality risks of antipsychotic medications in communitydwelling older adults. Br J Psychiatry 2014; 205: 44-51.

10 Kales HC, Kim HM, Zivin K, Valenstein M, Seyfried LS, Chiang C, et al. Risk of mortality among individual antipsychotics in patients with dementia. Am J Psychiatry 2012; 169: 71-9. 